Research Paper

\title{
Syndecan-1 Expression Is Associated with Tumor Size and EGFR Expression in Colorectal Carcinoma: A Clinicopathological Study of 230 Cases
}

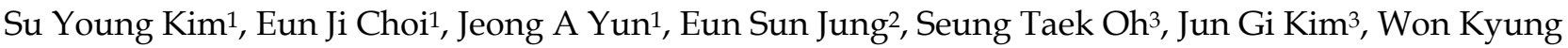 \\ Kang 3 , Sung Hak Lee ${ }^{2 \bowtie}$ \\ 1. Department of Pathology, The Catholic University of Korea, School of Medicine, Seocho-gu Banpodaero 222, Seoul 137-701, South Korea. \\ 2. Department of Hospital Pathology, The Catholic University of Korea, School of Medicine, Seocho-gu Banpodaero 222, Seoul 137-701, \\ South Korea. \\ 3. Department of Surgery, The Catholic University of Korea, School of Medicine, Seocho-gu Banpodaero 222, Seoul 137-701, South Korea.
}

$\triangle$ Corresponding author: Email: hakjjang@catholic.ac.kr; Tel: +82-2-2258-1617; Fax: +82-2-2258-1628

(C) Ivyspring International Publisher. This is an open-access article distributed under the terms of the Creative Commons License (http:/ / creativecommons.org/ licenses/by-nc-nd/3.0/). Reproduction is permitted for personal, noncommercial use, provided that the article is in whole, unmodified, and properly cited.

Received: 2014.09.06; Accepted: 2014.12.07; Published: 2015.01.01

\begin{abstract}
Background: Syndecan-1 (SDCl) is reported to modulate several key processes of tumorigenesis and has variable expression in many cancers. To date, the cause of altered expression has not been elucidated. In this study, we compared SDCl expression with various clinicopathological parameters and molecular markers to evaluate its clinical significance in colorectal carcinoma.

Methods: We screened for SDCl expression using immunohistochemistry in 230 surgical specimens of primary colorectal carcinoma from patients consecutively treated between 2008 and 2011 at Seoul St. Mary's Hospital, The Catholic University of Korea. The relationship between $\mathrm{SDCl}$ expression and various clinicopathological parameters and molecular markers was analyzed.

Results: The tumors were principally located in the left colon (71.3\%) and rectum (33.5\%). There were $216(93.9 \%)$ adenocarcinomas, 10 (4.3\%) mucinous adenocarcinomas, and 4 other tumors. Most of the carcinomas were pT3 $(68.3 \%)$ and PT4 (22.2\%). There was regional lymph node metastasis in 140 patients. SDCl expression was identified in the cancer cells of $212(96.8 \%)$ colon cancer cases. Of the SDCl-positive cases, 131 showed predominantly membranous immunopositivity, and 81 showed a predominantly cytoplasmic staining pattern. Mixed membranous and cytoplasmic staining was observed in 154 cases. In 93 cases, stromal SDCl reactivity was noted. Epithelial SDCl immunopositivity was significantly associated with tumor size $(p=0.016)$ and epidermal growth factor receptor expression $(p=0.006)$. However, it was not significantly correlated with lymph node metastasis, distant metastasis, lymphatic or vascular invasion, or KRAS mutation. In addition, stromal SDCl immunopositivity was significantly associated with the male $\operatorname{sex}(p=0.018)$.

Conclusions: The expression profile of SDCl may be of clinical value in colorectal cancer and may help in identifying aggressive forms of colorectal carcinoma. Further studies are needed in order to better understand the role of SDCl in the progression and invasiveness of colorectal carcinoma.
\end{abstract}

Key words: Syndecan-1, Expression, Colorectal cancer, Immunohistochemistry, Biomarkers

\section{Introduction}

Syndecans are type I transmembrane proteoglycans and have 3 major domains: an extracellular domain containing heparan sulfate chains, a trans- membrane domain, and a short cytoplasmic domain. Syndecan-1 (SDC1) is 1 of 4 cell surface heparan sulfate proteoglycans that are predominantly expressed 
by epithelial cells and plasma cells in adult tissues. Syndecans are involved in the regulation of cell-cell and cell-extracellular matrix (ECM) adhesion and cell migration; they mediate these processes in normal tissues through the binding of heparan sulfate chains to ECM molecules and other effectors, including growth factors, cytokines, proteinases, and proteinase inhibitors [1-3]. SDC1 can influence tumorigenesis by regulating the molecular mediators of tumor cell survival, proliferation, angiogenesis, and metastasis [1]. SDC1 expression is dysregulated in a number of cancers, including head and neck, ovarian, breast, and colorectal carcinomas [4-8].

The KRAS oncogene, a member of the ras family of oncogenes, is located at the chromosomal locus $12 \mathrm{p} 12$ and encodes a $21 \mathrm{kD}$ protein (p21ras) that is important in many guanosine triphosphate-coupled receptor tyrosine kinase signaling cascades, which modulate cellular proliferation and differentiation [9, 10]. KRAS mutations have been implicated in the development of diverse human malignancies and have been reported in pancreatic, ovarian, endometrial, biliary tract, lung, and colorectal cancers [11]. In colorectal cancer specimens, it has been reported that approximately $30-50 \%$ of cases harbor constitutive K-ras activation mutations, which principally occur in codons 12 and 13 [10, 12, 13]. Recently, KRAS mutations have been identified as being an important predictive marker for resistance to anti-epidermal growth factor receptor (EGFR) targeted therapy. Several studies have indicated that only colorectal cancers with wild-type K-ras expression respond to anti-EGFR treatments such as cetuximab and panitumumab [14-16].

In the present study, we evaluated the clinical implication of epithelial, cytoplasmic, and stromal SDC1 expression in colorectal cancer using immunohistochemistry. In addition, we analyzed the relationship between KRAS gene mutations and SDC1 expression.

\section{Materials and methods}

\section{Selection of patients and tumor samples}

A total of 230 patients ( 140 men and 90 women) with colorectal cancer who had undergone radical surgery at Seoul St. Mary's Hospital, The Catholic University of Korea between 2008 and 2011 were enrolled into this study. Clinicopathological parameters were reviewed retrospectively from the participants' medical records and pathology reports at our medical institution. The patients ranged between 32 and 93 (mean, 62.3) years of age. The mean tumor size was $4.85 \mathrm{~cm}$ (range, 0.7-17.0). Written informed consent was obtained from all patients. Patient consent and specimen collection were conducted in accordance with protocols approved by the Institutional Review Board of the Catholic University of Korea (KC13SISI0649).

\section{Tissue microarray (TMA) construction and immunohistochemistry}

After reviewing glass slides from the 230 cases of colorectal cancer, TMAs were constructed from paraffin-embedded blocks with a Manual Tissue Arrayer (Beecher Instruments, Sun Prairie, WI, USA) with a 2.0-mm tip. The TMAs were sectioned at a thickness of $4 \mu \mathrm{m}$. Sections from the TMA blocks were transferred to Probe On Plus slides (Fisher Scientific, Pittsburgh, PA, USA) and baked for 2 hours in a dry oven at $56^{\circ} \mathrm{C}$ (Agilent Technologies, Santa Clara, CA, USA). The sections were deparaffinized in xylene 3 times and rehydrated through $100 \%, 90 \%, 80 \%$, and $70 \%$ ethanol in Tris-buffered saline ( $\mathrm{pH} 7.4)$. The tissues were then boiled in $10 \mathrm{mM}$ sodium citrate buffer ( $\mathrm{pH}$ 6.0) using a microwave oven for $20 \mathrm{~min}$. After treating the tissues with $3 \% \quad \mathrm{H}_{2} \mathrm{O}_{2}$ in phosphate-buffered saline, the tissues were incubated with the diluted (1:50) anti-SDC1 mouse monoclonal antibody, B-A38 (Abcam, Cambridge, UK), at $4^{\circ} \mathrm{C}$ overnight. Having incubated the tissue with diluted (1:100) biotinylated anti-mouse antibody (Abnova, Walnut, CA, USA) for $1 \mathrm{~h}$ at room temperature, the signal was amplified using diluted ExtrAvidin-peroxidase (1:50; Sigma-Aldrich, St. Louis, $\mathrm{MO}, \mathrm{USA}$ ) for $1 \mathrm{~h}$ at room temperature. The liquid 3,3'-diaminobenzidine + Substrate Chromogen system (Dako, Glostrup, Denmark) was used for visualization. Membranous or cytoplasmic staining in cancer cells was deemed a positive result. The immunoreactivity of SDC1 was scored by adding the staining intensity ( 0 , no stain; 1 , weak; 2 , moderate; 3 , strong) to the points assigned based on the percentage of stained tumor cells present $(0$, no stain; $1,1-25 \% ; 2,26-50 \%$; $>50 \%$ ) for both membranous and cytoplasmic staining patterns. For the statistical analysis, we combined the membranous and cytoplasmic staining scores. Positivity for EGFR expression was defined as $>10 \%$ of tumor cells with any membrane staining above the background level. Cytoplasmic staining alone, without associated membrane staining, was considered negative, as in our previous study [17]. The immunohistochemical staining was independently scored by 2 pathologists.

\section{KRAS mutation test}

To prepare tissue samples, a hematoxylin and eosin-stained slide prepared from a colorectal cancer specimen was marked with a pen to indicate a tumor-rich area. The formalin-fixed, paraffin-embedded 
tissue blocks were then sectioned at a thickness of 10 $\mu \mathrm{m}$. In a subset of samples, tumor cells were scraped from glass slides with a scalpel under a dissecting microscope for DNA extraction. For deparaffinization, the scraped sections were incubated at room temperature in several volumes of xylene for 6 to $12 \mathrm{~h}$. For DNA extraction, deparaffinized tissue was digested with proteinase K (Qiagen, Inc., Valencia, CA) overnight at $37^{\circ} \mathrm{C}$. DNA was then isolated from the incubation mixture using a QIAcube robotic workstation (Qiagen Inc., Valencia, CA) extraction protocol. DNA yields were quantified using a Nanodrop spectrophotometer ND-1000 (Thermo Fisher Scientific Inc., Waltham, MA). The purified DNA samples were then tested using direct sequencing methods.

\section{Direct sequencing}

Approximately $60 \mathrm{ng}$ of genomic DNA prepared from formalin-fixed, paraffin-embedded tissue specimens was amplified using $10 \mathrm{pM}$ each of the KRAS forward and reverse primers (forward: RASO1 5'AAGGCCTGCTGAAAATGAC-3' and reverse: RASA2 5'-TGGTCCTGCACCAGTAATATG-3') and Taq polymerase PCR master mix (Promega Corporation, Madison, WI) in a $25 \mu \mathrm{L}$ reaction mixture. PCR was performed on an ABI 9700 thermocycler with 20 cycles using a touchdown protocol (starting annealing temperature of $65^{\circ} \mathrm{C}$, decreased $0.5^{\circ} \mathrm{C}$ per cycle) and 15 cycles with a $55^{\circ} \mathrm{C}$ annealing temperature. The resultant PCR products were purified using the QIAquick PCR Purification Kit (Qiagen Inc., Valencia, $\mathrm{CA}$ ) and the appropriate protocol on the QIAcube robotic workstation. The purified PCR products were sequenced in forward and reverse directions using an ABI 3730 automated sequencer (Applied Biosystems, Inc., Foster City, CA). Each chromatogram was visually inspected for any abnormalities, with particular attention being given to codons 12 and 13 .

\section{Statistical analysis}

The chi-square or Fisher's exact test was used to assess the association between SDC1 expression and various clinicopathological parameters. A p-value $<0.05$ was considered statistically significant. Data were analyzed using the SPSS statistical software version 21.0 (IBM, Armonk, NY) for Windows.

\section{Results}

The tumors were located in the right colon (including transverse colon) in $28.3 \%(65 / 230)$ of patients, in the left colon in $71.3 \%(164 / 230)$ of patients, and in the rectum in $33.5 \%(77 / 230)$ of patients. In one case, there was no information regarding the tumor site. SDC1 immunoreactivity was not significantly correlated with tumor location $(\mathrm{p}=0.735)$. There were 216 (93.9\%) adenocarcinomas, $10(4.3 \%)$ mucinous adenocarcinomas, and 4 other tumors. There were 8 cases $(3.5 \%)$ of well-differentiated carcinoma, 197 cases $(85.7 \%)$ of moderately differentiated carcinoma, and 13 cases $(5.7 \%)$ of poorly differentiated carcinoma. In 12 cases, the tumor differentiation status was unavailable. Regarding the primary tumor stage, 2 $(0.8 \%)$ cases were pT1, $14(6.1 \%)$ were pT2, 157 (68.3\%) were pT3, and $51(22.2 \%)$ were pT4. Tumor stage was not available for 6 cases. Regional lymph node metastasis was noted in 140 cases $(60.9 \%)$.

In the normal colonic mucosa, SDC1 is expressed at the basolateral membrane of the crypt epithelium and in the plasma cells of the lamina propria (Fig. 1A). In the colon cancer specimens, SDC1 staining results were available in 219 cases. Of these, positive SDC1 immunoreactivity was identified in the cancer cells of 212 cases $(96.8 \%)$ of colon cancer. Of the SDC1-positive cases, 131 predominantly showed membranous immunopositivity, and 81 cases showed a predominantly cytoplasmic staining pattern. Exclusively cytoplasmic or membranous staining was observed in 28 and 30 cases, respectively. There were 154 cases, which showed a mixed membranous and cytoplasmic staining pattern (Fig. 1B-1E). In 93 cases, stromal SDC1 reactivity was noted (Fig. 1F). Epithelial SDC1 immunopositivity was significantly associated with an advanced primary tumor ( $\mathrm{T}$ stage; $\mathrm{p}=0.016$ ) and EGFR immunohistochemical positivity $(\mathrm{p}=$ 0.006). In contrast, SDC1 expression was not significantly correlated with lymph node metastasis, distance metastasis, lymphatic or vascular invasion, or KRAS mutation states. Stromal SDC1 immunopositivity was significantly associated with the male sex (p $=0.018$ ) and marginally associated with distant metastasis $(p=0.072)$. These findings are summarized in Tables 1 and 2. In addition, in the epithelial SDC1 immunopositivity cases, we evaluated which expression patterns, namely membranous or cytoplasmic, were significantly associated with various clinicopathological or molecular parameters. Membranous SDC1 immunopositivity, including a predominant and exclusive expression pattern, was significantly associated with advanced primary tumors $(p=0.001)$ and EGFR immunohistochemical positivity $(\mathrm{p}=$ 0.016). Moreover, membranous SDC1 immunoreactivity was significantly associated with stromal SDC1 immunohistochemical positivity $(p=0.021)$. In contrast, membranous or cytoplasmic SDC1 expression was not significantly correlated with other clinicopathological parameters or the KRAS mutation state (Table 3). 


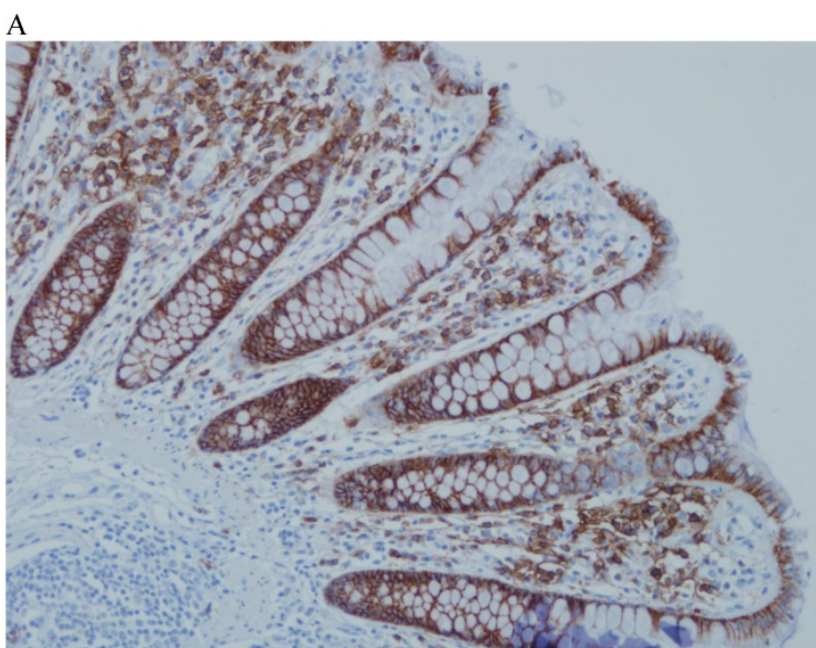

\section{B}
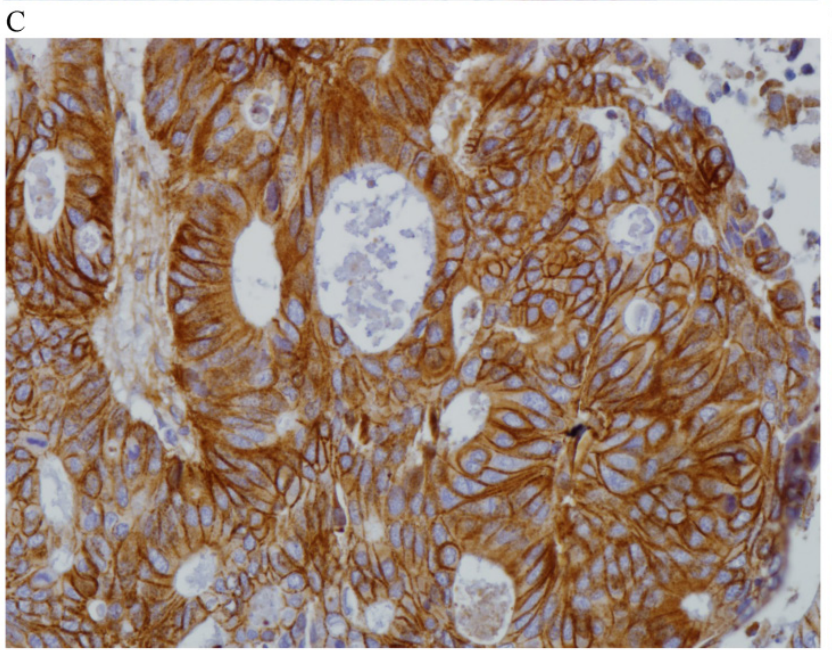

E
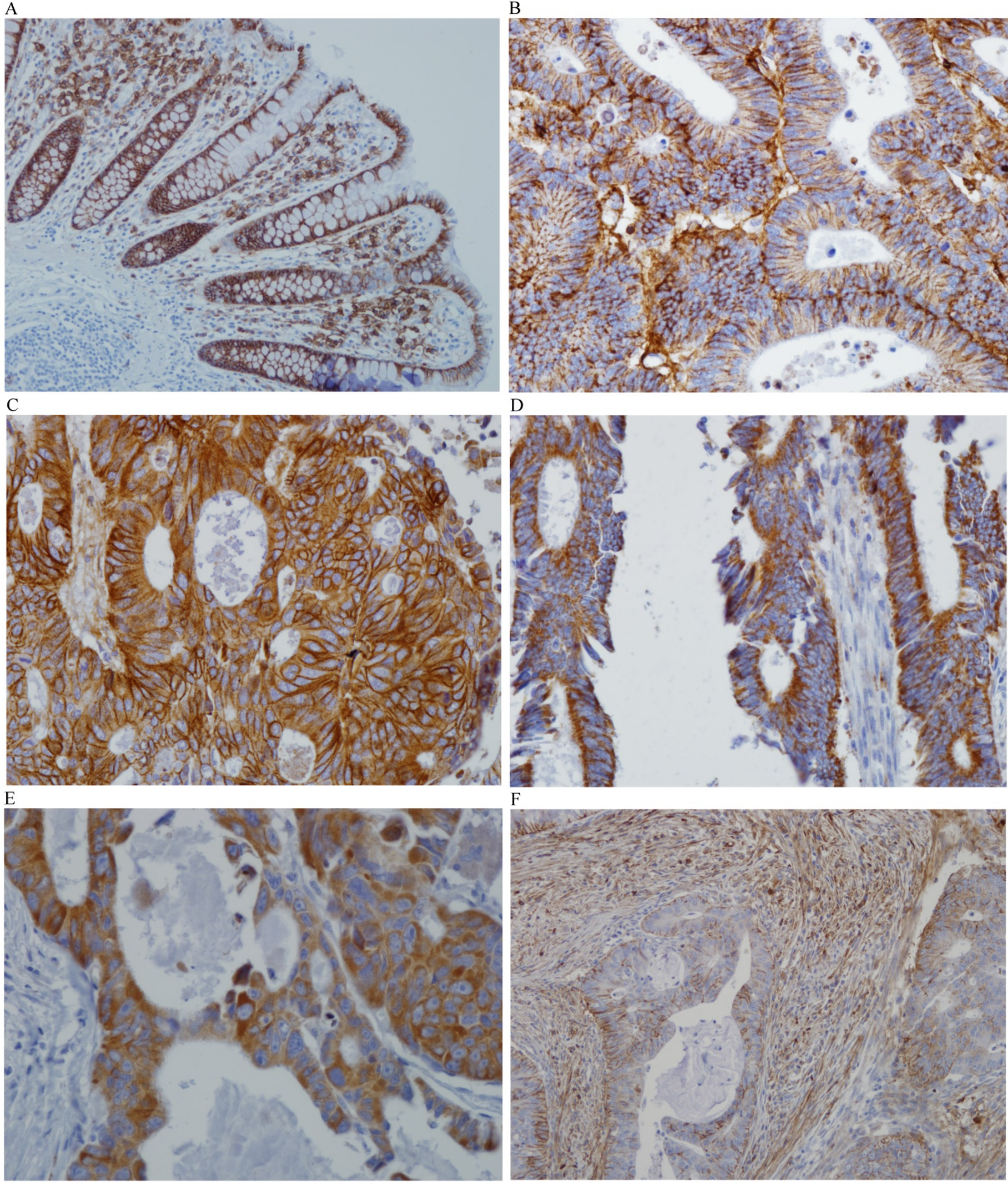

$\mathrm{D}$

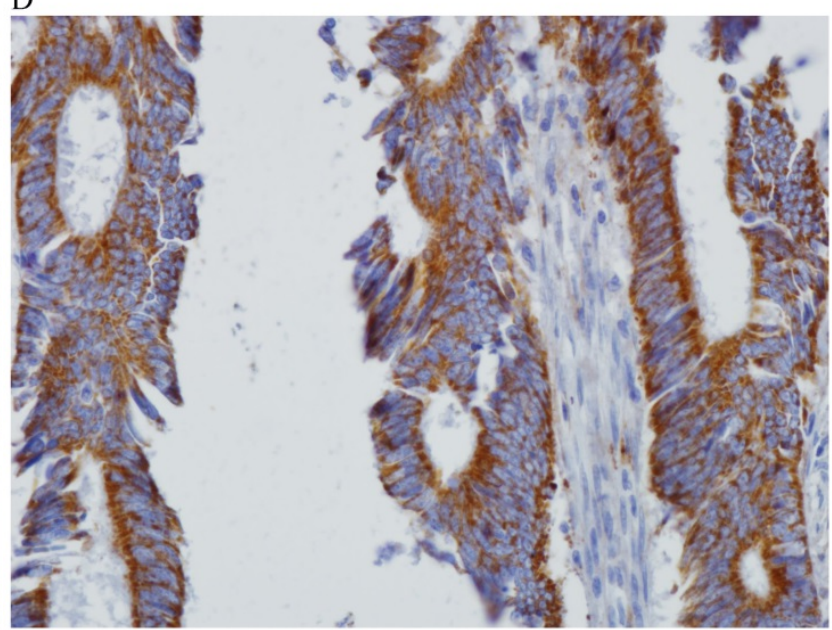

$\mathrm{F}$

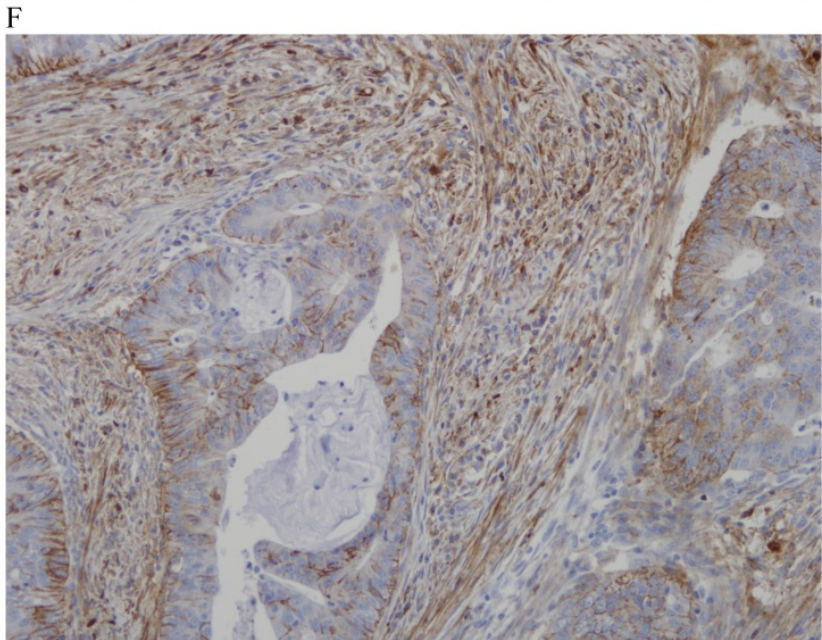

Fig. 1. Representative syndecan-1 (SDCl) immunohistochemical staining in $(\mathbf{A})$ normal colonic mucosa $(\times 200)$ and colorectal carcinoma with $($ B) only membranous staining ( $\times 400),(C)$ predominantly membranous staining $(\times 400)$, (D) only cytoplasmic staining ( $\times 400)$, and (E) predominantly cytoplasmic staining $(\times 400)$ staining patterns. $(\mathbf{F})$ A case showing SDC1 immunopositivity in the stromal spindle cell component of tumor nests $(\times 200)$. 
Table 1. The relationship between syndecan-1 (SDC1) expression and the clinicopathological parameters of patients with colorectal carcinomas

\begin{tabular}{|c|c|c|c|c|c|c|}
\hline Parameter & eSDC1 (n & & p-value & sSDC1 ( & & p-value \\
\hline & Positive ${ }^{b}$ & Negative & & Positive & Negative & \\
\hline Sex & & & 0.131 & & & $0.018^{*}$ \\
\hline Male & 42 & 94 & & 48 & 92 & \\
\hline Female & 20 & 63 & & 45 & 45 & \\
\hline Age & & & 0.596 & & & 0.192 \\
\hline$\leq 55$ years & 14 & 42 & & 20 & 40 & \\
\hline$>55$ years & 48 & 115 & & 73 & 97 & \\
\hline Tumor stage $c$ & & & $0.016^{*}$ & & & 0.427 \\
\hline $\mathrm{T} 1-2$ & 6 & 11 & & 6 & 11 & \\
\hline T3-4 & 55 & 143 & & 86 & 121 & \\
\hline Nodal stage & & & 0.561 & & & 0.867 \\
\hline N0 & 24 & 60 & & 37 & 53 & \\
\hline N1-2 & 38 & 97 & & 56 & 84 & \\
\hline Metastasis & & & 0.428 & & & 0.072 \\
\hline M0 & 58 & 144 & & 89 & 122 & \\
\hline M1 & 4 & 13 & & 4 & 15 & \\
\hline Lymphatic invasion & & & 0.143 & & & 0.918 \\
\hline Absent & 24 & 54 & & 34 & 51 & \\
\hline Present & 38 & 103 & & 59 & 86 & \\
\hline Vascular invasion & & & 0.650 & & & 0.754 \\
\hline Absent & 56 & 142 & & 83 & 124 & \\
\hline Present & 6 & 15 & & 10 & 13 & \\
\hline Perineural invasion & & & 0.513 & & & 0.707 \\
\hline Absent & 42 & 111 & & 63 & 96 & \\
\hline Present & 20 & 46 & & 30 & 41 & \\
\hline Differentiation $^{\mathrm{d}}$ & & & 0.505 & & & 0.986 \\
\hline Well-to-moderate & 57 & 139 & & 83 & 122 & \\
\hline Poor & 1 & 11 & & 5 & 8 & \\
\hline
\end{tabular}

Table 2. Relationship between SDCl expression and epidermal growth factor receptor expression, and KRAS mutation status

\begin{tabular}{|c|c|c|c|c|c|c|}
\hline \multirow[t]{2}{*}{ Marker } & \multicolumn{2}{|c|}{ eSDC1 (n = 219) } & \multirow[t]{2}{*}{ p-value } & \multicolumn{2}{|c|}{ sSDC1 $(n=230)$} & \multirow[t]{2}{*}{ p-value } \\
\hline & Positive & Negative & & Positive & Negative & \\
\hline KRAS & & & 0.293 & & & 0.328 \\
\hline Positive & 22 & 70 & & 42 & 53 & \\
\hline Negative & 40 & 87 & & 51 & 84 & \\
\hline EGFR & & & $0.006^{*}$ & & & 0.245 \\
\hline Positive & 51 & 111 & & 71 & 95 & \\
\hline Negative & 11 & 46 & & 22 & 42 & \\
\hline
\end{tabular}

eSDC1, epithelial syndecan-1 immunohistochemical staining; sSDC1, stromal syndecan-1 immunohistochemical staining; EGFR, epidermal growth factor receptor.

* Statistically significant

\section{Discussion}

The present study demonstrates that SDC1 expression in cancer cells is significantly correlated with tumor aggressiveness. However, in previous studies of colorectal cancer, loss of epithelial SDC1 expression has been shown to be associated with an advanced
Table 3. Relationship between SDCl expression pattern and epidermal growth factor receptor expression, and KRAS mutation status

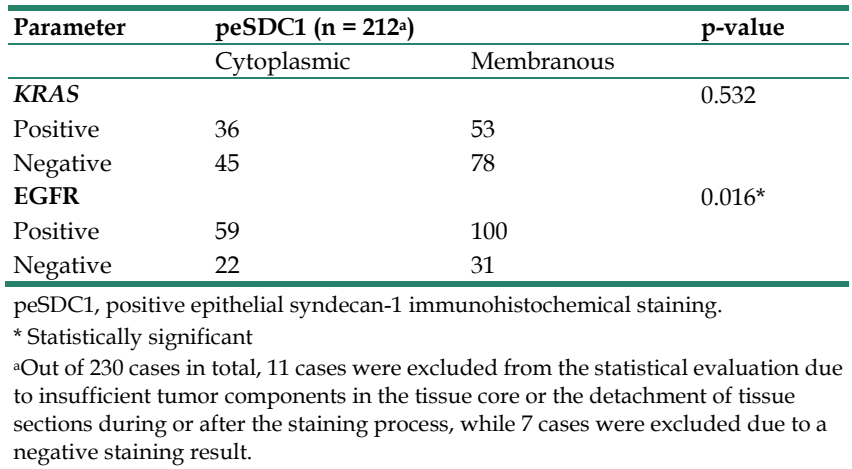

clinical stage and poor patient prognosis [4, 5]. Several theories have been proposed to account for the observed association between reduced epithelial SDC1 expression and tumor progression. Cell surface SDC1 is thought to enhance cell-ECM cohesion and restrict cell migration. Thus, the loss of epithelial SDC1 increases the migratory capacity of tumor cells [1]. In 
addition, release of the SDC1 ectodomain from the cell surface could play an important role. The extracellular domain of SDC1 can bind to diverse signaling proteins and growth factors, such as the transforming growth factor and fibroblast growth factor, which can affect tumor progression. Thus, shedding of the ectodomain could disrupt SDC1-signaling protein linkage, releasing the growth factors, which would serve to promote the proliferation of cancer cells [1, 18]. Furthermore, epithelial-to-mesenchymal transition, in which invasive cancer cells change from an epithelial to a less-differentiated mesenchymal phenotype, is a key process in tumor progression. An absence of SDC1 epithelial expression is a hallmark of epithelial-to-mesenchymal transition. Thus, the loss of epithelial SDC1 is associated with a biologically more aggressive phenotype and a worse clinical outcome $[4,19,20]$.

However, unlike the present study, previous reports on SDC1 expression in colorectal carcinoma have only examined membranous and stromal staining in carcinoma cells when assessing immunohistochemical staining results as positive or negative $[4,5]$. In this study, we assessed SDC1 expression in abnormal tumor cell locations; we scored the cytoplasmic as well as membranous expression of SDC1, as previously reported [6]. Using this scoring scheme, we found that SDC1 immunopositivity was associated with a poor prognosis, which is consistent with the results of our previous study [6]. This may partly explain the differences between our results and those of previous studies. In the present study, we showed that the normal membranous pattern of SDC1 expression is disrupted in tumor cells, and increased amounts of SDC1 were identified in the cytoplasm of tumor cells in 81 cases. This translocation of SDC1 from the cell membrane to the cytoplasm of the tumor cells is anticipated to result in a low level of functional SDC1 on the cell surface. This may result in the tumor cells having fewer ECM interactions, and thus allow them to move more freely, leading to invasion and distant metastasis. Considering these findings, it is likely that increased cytoplasmic expression of SDC1 may result in a decrease in effective SDC1 protein on the cell surface or conceal decreased SDC1 expression on the cell surface, when cytoplasmic and membranous expressions are interpreted to be equivalent. Thus, high levels of SDC1 expression do not necessarily equate with a significant amount of functional SDC1 protein. In addition, we show that among epithelial SDC1 immunopositivity cases, membranous rather than cytoplasmic SDC1 immunopositivity was significantly associated with advanced primary tumors and EGFR immunohistochemical positivity. Teng et al. suggested that membrane-bound and sol- uble forms of SDC1 may play unique roles at different stages of cancer progression [1]. Furthermore, in certain malignancies such as gallbladder and thyroid cancer, SDC1 expression has been reported to be associated with unfavorable prognosis [21, 22]. Taking these facts together, different SDC1 expression in diverse cancer types suggests that the role of SDC1 can be affected by the underlying cancer type, which may explain the discrepancies in SDC1 expression and prognosis in different cancers. In addition, to the best of our knowledge, there has been no report about mutations in highly conserved regions of SDC1 (i.e., transmembrane and cytoplasmic domains). Thus, it is thought that distinct SDC1 expression may not be derived from SDC1 mutation. Further studies are required to understand the effect of altered cytoplasmic and membranous SDC1 expression on tumorigenesis.

In $40.4 \%(93 / 230)$ of the specimens, stromal staining for SDC1 was observed. This proportion is slightly lower than that in a previous study by Lundin et al., in which stromal SDC1 immunoreactivity was noted in $58 \%$ of the specimens [5]. In the study conducted by Lundin et al., no statistically significant association between stromal SDC1 immunoreactivity and various clinicopathological parameters was identified [5]. However, in the present study, stromal SDC1 immunoreactivity was significantly associated with the male sex $(p=0.018)$ and marginally associated with distant metastasis $(p=0.072)$. To the best of our knowledge, there is no report of statistically significant association between stromal SDC1 expression and sex in various types of cancer. The reasons for the increase in stromal SDC1 expression in male patients with colorectal cancer are not readily evident. Further larger and more long-term studies should clarify this role. Meanwhile, it is unclear whether stromal SDC1 protein originates from ectodomain shedding from the tumor cell membrane or from within the stromal tissue itself [23]. The tumor microenvironment provides a compatible niche for the growth and progression of tumor cells, and stromal SDC1 may influence the tumor microenvironment by altering ECM-cytoskeleton linkage in the vicinity of the tumor [1]. It has recently been shown that the amount of stromal SDC1 protein can be increased by epithelial-mesenchymal interaction and is related to tumor progression and/or metastasis in several cancers [19, 24]. Ito et al. suggested that stromal SDC1 is secreted by cancer cells and entrapped by stroma cells, but when excessive SDC1 is produced from cancer cells, the remainder may still be deposited in cancer cells after shedding, which leads to some stimulation related to cancer progression [21].

In addition, we reveal that stromal SDC1 immunoreactivity is significantly associated with mem- 
branous SDC1 expression, which is significantly correlated with advanced primary tumors and EGFR immunohistochemical positivity. At present, it is difficult to elicit how stromal and epithelial SDC1 expressions are related. Further studies with different datasets are required to validate the statistical and prognostic significance of stromal SDC1 immunoreactivity.

Our study indicated that SDC1 expression was not significantly correlated with KRAS mutation status. Until recently, few studies have investigated the relationship between SDC1 expression and KRAS mutation states. Vuoriluoto et al. demonstrated that activating KRAS mutations are correlated with the increased expression of a2 $\beta 1$ integrin, membrane type-1 matrix metalloproteinase (MT1-MMP), and SDC1 [25]. Several studies have indicated that MT1-MMP and a2 $\beta 1$ integrin are important regulators of tumor cell invasion into the collagen matrix [1, 26 , 27]. Vuoriluoto et al. showed that strong SDC1 expression was inversely correlated with MT1-MMP expression; upon decreased SDC1 expression, MT1-MMP-dependent single-cell invasion into the collagen matrix occurred [25]. Vuoriluoto et al. also demonstrated that KRAS mutation is important for a2 $\beta 1$ integrin and MT1-MMP-dependent invasion into collagen [25]. In line with these findings, low SDC1 expression was shown to be correlated with a worse prognosis in patients with colorectal cancers [4, 5]. However, previous studies have reported that high levels of SDC1 are linked to a poor prognosis in several cancer types $[6,20,28]$. Vuoriluoto et al. suggested that this discrepancy might stem from different biological properties between membrane-bound and soluble-shed ectodomain forms of SDC1, and these 2 forms may not be distinguished by immunohistochemical staining [25]. They also suggested that the SDC1 ectodomain alone might function as an invasion enhancer, whereas the membrane-bound full-length receptor may be able to exert MT1-MMP inhibitory signaling by previously unknown mechanisms. The reason that the association between SDC1 staining and colorectal carcinoma aggressiveness in our study cohort is opposite to the relationship identified in other studies requires further clarification.

We also examined the relationship between SDC1 and EGFR, a widely used prognostic factor for colorectal carcinoma. There was a significant association between SDC1 immunostaining on tumor cells and increased EGFR staining. Few studies have evaluated the association between SDC1 and EGFR. Shah et al. found that immunopositivity for SDC1 and EGFR were both significantly correlated with a favorable prognosis for patients with non-small cell lung carcinoma [29]. However, they did not investi- gate the relationship between these biological markers. Gialeli et al. demonstrated that panitumumab, a selective inhibitor of EGF-induced EGFR activation, can prevent the expression of matrix effectors, such as MT1-MMP and syndecan-4, resulting in a synergistic effect with therapeutic strategies [30]. Although the molecular events underlying the interactions between SDC1 and EGFR remain to be elucidated, it can be inferred that these 2 markers are related [30].

In conclusion, we have shown that epithelial SDC1 immunopositivity is significantly correlated with primary tumor stage and EGFR immunohistochemical reactivity. In addition, stromal SDC1 immunopositivity was significantly associated with male sex and marginally associated with distant metastasis. These findings may help to identify aggressive forms of colorectal carcinoma. The association between SDC1 protein expression and the outcome in patients with colorectal cancer may elucidate the role of SDC1 in the progression and invasiveness of colorectal carcinoma, which could lead to the development of novel therapeutic agents.

\section{Abbreviations}

ECM: extracellular matrix; EGFR: epidermal growth factor receptor; MT1-MMP: membrane type-1 matrix metalloproteinase; SDC1: syndecan-1; TMA: tissue microarray

\section{Acknowledgments}

This research was supported by the Basic Science Research Program through the National Research Foundation of Korea, as funded by the Ministry of Education, Science, and Technology (2012R1A1A2042078).

\section{Authors' contributions}

SHL and SYK conceived the study, performed the statistical analysis, and drafted the manuscript. EJC and JAY carried out the molecular pathological studies. ESJ carried out the pathological assessments. STO collected the samples and performed some of the experiments. All authors have read and approved the final manuscript.

\section{Competing Interests}

The authors have declared that no competing interest exists.

\section{References}

1. Teng YH, Aquino RS, Park PW. Molecular functions of syndecan-1 in disease. Matrix Biol. 2012; 31: 3-16.

2. Tkachenko E, Rhodes JM, Simons M. Syndecans: new kids on the signaling block. Circ Res. 2005; 96: 488-500.

3. Lambaerts K, Wilcox-Adelman SA, Zimmermann P. The signaling mechanisms of syndecan heparan sulfate proteoglycans. Curr Opin Cell Biol. 2009; 21: 662-9. 
4. Hashimoto Y, Skacel M, Adams JC. Association of loss of epithelial syndecan-1 with stage and local metastasis of colorectal adenocarcinomas: an immunohistochemical study of clinically annotated tumors. BMC cancer. 2008; 8: 185.

5. Lundin M, Nordling S, Lundin J, Isola J, Wiksten JP, Haglund C. Epithelial syndecan-1 expression is associated with stage and grade in colorectal cancer. Oncology. 2005; 68: 306-13.

6. Lee SH, Choi EJ, Kim MS, et al. Prognostic significance of syndecan-1 expression in squamous cell carcinoma of the tonsil. Int J Clin Oncol. 2014; 19: 247-53.

7. Lendorf ME, Manon-Jensen T, Kronqvist P, Multhaupt HA, Couchman JR. Syndecan-1 and syndecan-4 are independent indicators in breast carcinoma. J Histochem Cytochem. 2011; 59: 615-29.

8. Kusumoto T, Kodama J, Seki N, Nakamura K, Hongo A, Hiramatsu Y. Clinical significance of syndecan-1 and versican expression in human epithelial ovarian cancer. Oncol Rep. 2010; 23: 917-25.

9. Vogelstein B, Fearon ER, Hamilton SR, et al. Genetic alterations during colorectal-tumor development. N Engl J Med. 1988; 319: 525-32.

10. Bolocan A, Ion D, Ciocan DN, Paduraru DN. Prognostic and predictive factors in colorectal cancer. Chirurgia. 2012; 107: 555-63.

11. Sacco E, Spinelli M, Vanoni M. Approaches to Ras signaling modulation and treatment of Ras-dependent disorders: a patent review (2007-present). Expert Opin Ther Pat. 2012; 22: 1263-87.

12. Andreyev HJ, Norman AR, Cunningham D, Oates JR, Clarke PA. Kirsten ras mutations in patients with colorectal cancer: the multicenter "RASCAL" study. J Natl Cancer Inst. 1998; 90: 675-84.

13. Boughdady IS, Kinsella AR, Haboubi NY, Schofield PF. K-ras gene mutations in adenomas and carcinomas of the colon. Surg Oncol. 1992; 1: 275-82.

14. Petrelli F, Borgonovo K, Cabiddu M, Ghilardi M, Barni S. Cetuximab and panitumumab in KRAS wild-type colorectal cancer: a meta-analysis. Int J Colorectal Dis. 2011; 26: 823-33.

15. Lievre A, Bachet JB, Boige V, et al. KRAS mutations as an independent prognostic factor in patients with advanced colorectal cancer treated with cetuximab. J Clin Oncol. 2008; 26: 374-9.

16. Amado RG, Wolf M, Peeters $M$, et al. Wild-type KRAS is required for panitumumab efficacy in patients with metastatic colorectal cancer. J Clin Oncol. 2008; 26: 1626-34.

17. Lee SH, Lee YS, Hong YG, Kang CS. Significance of COX-2 and VEGF expression in histopathologic grading and invasiveness of meningiomas. APMIS. 2014; 122: 16-24.

18. Choi S, Lee H, Choi JR, Oh ES. Shedding; towards a new paradigm of syndecan function in cancer. BMB Rep. 2010; 43: 305-10.

19. Contreras HR, Ledezma RA, Vergara J, et al. The expression of syndecan-1 and -2 is associated with Gleason score and epithelial-mesenchymal transition markers, E-cadherin and beta-catenin, in prostate cancer. Urol Oncol. 2010; 28: 534-40. doi:10.1016/j.urolonc.2009.03.018.

20. Loussouarn D, Campion L, Sagan C, et al. Prognostic impact of syndecan-1 expression in invasive ductal breast carcinomas. Br J Cancer. 2008; 98: 1993-8.

21. Ito $\mathrm{Y}$, Yoshida H, Nakano K, et al. Syndecan-1 expression in thyroid carcinoma: stromal expression followed by epithelial expression is significantly correlated with dedifferentiation. Histopathology. 2003; 43: 157-64.

22. Roh YH, Kim YH, Choi HJ, Lee KE, Roh MS. Syndecan-1 expression in gallbladder cancer and its prognostic significance. Eur Surg Res. 2008; 41: 245-50.

23. Bayer-Garner IB, Dilday B, Sanderson RD, Smoller BR. Syndecan-1 expression is decreased with increasing aggressiveness of basal cell carcinoma. Am J Dermatopathol. 2000; 22: 119-22.

24. Vered M, Dayan D, Yahalom R, et al. Cancer-associated fibroblasts and epithelial-mesenchymal transition in metastatic oral tongue squamous cell carcinoma. Int J Cancer. 2010; 127: 1356-62.

25. Vuoriluoto K, Hognas G, Meller P, Lehti K, Ivaska J. Syndecan-1 and -4 differentially regulate oncogenic K-ras dependent cell invasion into collagen through alpha2beta1 integrin and MT1-MMP. Matrix Biol. 2011; 30: 207-17.

26. Li XY, Ota I, Yana I, Sabeh F, Weiss SJ. Molecular dissection of the structural machinery underlying the tissue-invasive activity of membrane type- 1 matrix metalloproteinase. Mol Biol Cell. 2008; 19: 3221-33.

27. Sabeh F, Ota I, Holmbeck K, et al. Tumor cell traffic through the extracellular matrix is controlled by the membrane-anchored collagenase MT1-MMP. J Cell Biol. 2004; 167: 769-81.

28. Barbareschi M, Maisonneuve $\mathrm{P}$, Aldovini D, et al. High syndecan-1 expression in breast carcinoma is related to an aggressive phenotype and to poorer prognosis. Cancer. 2003; 98: 474-83.

29. Shah L, Walter KL, Borczuk AC, et al. Expression of syndecan-1 and expression of epidermal growth factor receptor are associated with survival in patients with nonsmall cell lung carcinoma. Cancer. 2004; 101: 1632-8.

30. Gialeli C, Theocharis AD, Kletsas D, Tzanakakis GN, Karamanos NK. Expression of matrix macromolecules and functional properties of EGF-responsive colon cancer cells are inhibited by panitumumab. Invest New Drugs. 2013; 31: 516-24. 